\title{
Stationary Distribution of Random Motion with Delay in Reflecting Boundaries*
}

\author{
Anatoliy A. Pogorui ${ }^{1}$, Ramón M. Rodríguez-Dagnino ${ }^{2}$ \\ Tecnológico de Monterrey (ITESM), Electrical and Computer Engineering. Sucursal de correos, Monterrey, México. \\ E-mail: \{pogorui,rmrodrig\}@itesm.mx \\ Received February 21, 2010; revised April 26, 2010; accepted May 8, 2010
}

\begin{abstract}
In this paper we study a continuous time random walk in the line with two boundaries $[a, b], a<b$. The particle can move in any of two directions with different velocities $v_{1}$ and $v_{2}$. We consider a special type of boundary which can trap the particle for a random time. We found closed-form expressions for the stationary distribution of the position of the particle not only for the alternating Markov process but also for a broad class of semi-Markov processes.
\end{abstract}

Keywords: Random Motion, Reflecting Boundaries, Semi-Markov, Random Walk

\section{Introduction}

In this paper we study the stationary distribution of a one-dimensional random motion performed with two velocities, where the random times separating consecutive velocity changes perform an alternating Markov process. The sojourn times of this process are exponentially distributed random variables. There are many papers on random motion devoted to analysis of models in which motions are driven by a homogeneous Poisson process [1-4], however we have not found any paper investigating the stationary distribution of these processes.

We assume that the particle moves on the line $\mathbb{R}$ in the following manner: At each instant it moves according to one of two velocities, namely $v_{1}>0$ or $v_{2}<0$ Starting at the position $x_{0} \in \mathbb{R}$ the particle continues its motion with velocity $v_{1}>0$ during random time $\tau_{1}$, where $\tau_{1}$ is an exponential random variable with parameter $\lambda_{1}$, then the particle moves with velocity $v_{2}<0$ during random time $\tau_{2}$, where $\tau_{2}$ is an exponential distributed random variable with parameter $\lambda_{2}$. Furthermore, the particle moves with velocity $v_{1}>0$ and so on. When the particle reaches boundary $a$ or $b$ it will stay at that boundary a random time given by the time the particle remains in the same direction up to the time such a particle changes direction. Similar partly reflecting (or trapping) boundaries have been considered in [5], and they may be found in optical photon propagation in

*We thank ITESM through the Research Chair in Telecommunications. turbid medium or chemical processes with sticky layers or boundaries.

We also consider a generalization of these results for semi-Markov processes, i.e., when the random variables $\tau_{1}$ and $\tau_{2}$ are different from exponential. This paper is divided in two main parts, namely the Markov case and the generalization to the semi-Markov modeling. Our main result, in the first part of this paper, consists on finding the stationary distribution of the well-known telegrapher process on the line with delays in reflecting boundaries. In the second part, we find the stationary distribution of a more general continuous time random walk when the sojourn times are generally distributed.

\section{Markov Case}

\subsection{Mathematical Modeling}

Let us set the probability space $(\Omega, \mathcal{F}, \mathrm{P})$. On the phase space $\mathbf{E}=\{1,2\}$ consider an alternating Markov process $\{\xi(t) ; t \geq 0\}$ having the sojourn time $\tau_{i}$ corresponding to the state $i \in \mathbf{E}$, and transition probability matrix of the embedded

Markov chain

$$
P=\left[\begin{array}{ll}
0 & 1 \\
1 & 0
\end{array}\right]
$$

Denote by $\{x(t) ; t \geq 0\}$ the position of the particle at time $t$. Consider the function $C(x)$ on the space $\mathbf{E}$ which is defined as 


$$
C(x)=\left\{\begin{array}{lll}
v_{1} & \text { if } & x=1 \\
v_{2} & \text { if } & x=2 .
\end{array}\right.
$$

The position of the particle at any time $t$ can be expressed as

$$
x(t) \xi x_{0} d s \int_{0}^{t} C(()),
$$

where the starting point $x_{0} \in[a, b]$.

Equation (3) determines the random evolution of the particle in the alternating Markov medium $\{\xi(t) ; t \geq$ $0\}$ [6,7]. So, $x(t)$ is the well-known one-dimensional telegraph process $[1,2]$. We assume that $a<b$ are two delaying or adhesive boundaries on the line such that if a particle reaches boundary $a$ or $b$ then it is delayed until the instant that the process changes velocity.

Now, consider the two-component stochastic process $\left\{\zeta t\left(\xi_{t=x} t(),()\right\}\right.$ on the phase space $\mathbb{Z}=[a, b]$ $\times\{1,2\}$. The process $\zeta t()$ is a homogeneous Markov process with the following generating operator $[6,7]$ :

$A \phi(x, i) \nexists \mathbb{R C}(x, i i) \frac{d}{d x} \phi(x x i i)+i{ }_{i}[\phi()-,\phi()],,=1,2$,

where $P \phi(x, 1)=\phi(x, 2)$ and $P \phi(x, 2)=\phi(x, 1)$.

\subsection{Stationary Distribution}

Denote by $\pi(\cdot)$ the stationary distribution of $\zeta(t)$. The analysis of the properties of the process $\zeta(t)$ leads up to the conclusion that the stationary distribution $\pi$ has atoms at points $(a, 2)$ and $(b, 1)$, and we denote them as $\pi[a, 2]$ and $\pi[k, 1]$ respectively. The continuous part of $\pi$ is denoted as $\pi(i,) ;, \in \mathbf{E}$.

Since $\pi$ is the stationary distribution of $\zeta \pi($ ) then for any function $\phi(\cdot)$ from the domain of the operator $A$ we have

$$
\int_{\mathbb{Z}} A \phi(\pi z) d()=0
$$

Now, let $A^{*}$ be the conjugate or adjoint operator of $A$. Then by changing the order of integration in (5) (integrating by parts), we can obtain the following expressions for the continuous part of $A^{*} \pi=0$

$$
\left\{\begin{array}{l}
\underset{v_{1} x \times \lambda \pi x\left((\pi x)+{ }_{1}\right.}{d x}(, 1)-_{2}(, 2)=0 \\
v_{2 \pi x \lambda \pi x}^{d} d x
\end{array}\right.
$$

Similarly, from (5) we obtain

$$
\left\{\begin{array}{l}
\lambda_{\mathrm{T}} \pi b(\pi, h) *_{1}\left({ }^{-}, 1\right)=0 \\
\lambda_{\mathrm{T}} \pi b(\pi, h) *_{2}\left({ }^{-}, 2\right)=0 \\
\lambda_{2} \pi a(\pi, \Omega) \forall_{2}\left({ }^{+}, 2\right)=0 \\
\lambda_{2} \pi a(\pi, \Omega) \forall_{1}\left({ }^{+}, 1\right)=0
\end{array}\right.
$$

where $\pi \phi \bar{\pi} \dot{x}, i):=\lim _{x \uparrow b}($,$\left.) and \pi \phi{ }^{+} i,\right):=\lim _{x \downarrow a}$ $\pi x(i$,$) for i=1,2$.

It follows from the set (6) that

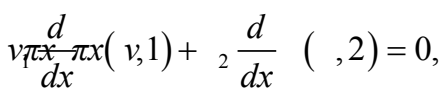

or equivalently $v \pi x(\pi, \mathrm{d}){ }_{t}{ }_{2} k(, 2)==$ constant

By using (7), we get $v_{p} b b(\pi b, 1) * v_{2} b\left({ }^{-}, 2\right)=0$, consequently $k=0$ and

$$
v \pi x(\pi, d) H_{2}(, 2)=0
$$

for all $x \in[a, b]$.

By obtaining $\pi x(2)$ from (8) and substituting such a result into the first equation in the set (6) we have

$$
\underset{v_{1} \pi x \lambda \pi x(2 \pi x)-{ }_{1} \cdot(, 1)-{ }_{2} \frac{v_{1}}{v_{2}}}{d x}(, 1)=0
$$

Solving (9) we obtain for the continuous part of $\pi$

$$
\pi x(1) € e^{-\mu x}
$$

And

$$
\pi(, 2)=\epsilon \frac{v_{1}}{v_{2}}-\mu x
$$

where $\mu .=\frac{\lambda \lambda}{v_{1}}+\frac{2}{v_{2}}$

Now, from (7) we obtain for atoms

$$
\pi[a, 2]=\frac{v_{1}}{\lambda_{2}} C e^{-\mu a}
$$

and

$$
\pi k, 1]=\frac{v_{1}}{\lambda_{1}} C e^{-\mu b}
$$

The factor $C$ can be calculated from the normalization equation

$$
\int_{\mathbb{Z}} \pi \notin d z=1
$$

or equivalently

$$
\left.\int_{a}^{b} \pi x, \pi\right) d x d d x b(, 2)+[, 2]+[, 1]=1
$$

It follows from (15) that

$\left.C=\left[\left(\frac{v_{1}}{\lambda \mu \nu \lambda \mu \nu} \frac{1}{2}\right) v_{2}-v_{1}\right)+\left(\frac{v_{1}}{2}-\frac{1}{2} \frac{v_{1}-v_{2}}{2}\right) e^{-}\right]^{-1}$. 
We should notice that the stationary distribution $\bar{\pi}(x)$ of the process $x(t)$ over the interval $(a, b)$ is $\bar{\pi}(x)$ $=\pi x(\pi x 1)+. \quad(, 2)$

\subsection{Balanced and One-Boundary Cases}

\subsubsection{Balanced Case}

Let us call the balanced case when $\mu .=\frac{\lambda \lambda}{v_{1}}+\frac{2}{v_{2}}=0$ In this case we can observe that $\pi(x, 1)$ and $\pi(2)$ do not depend on $x$. Hence, the continuous part of the stationary distribution of the process $x(t)$ is uniform over the open interval $(a, b)$. Now, the factor $C$, say $C_{B}$, reduces to

$$
C_{B}=\frac{v_{2}}{\left(v_{2}-v_{1}\right)(b-a+)},
$$

where $\delta .=\frac{v_{1}}{\lambda \lambda}=-\frac{v_{2}}{2}$

Therefore, the stationary distribution can be expressed as

$$
\left.\pi(x, 3 x) €_{B} \text { and } \mathbb{C}, 2\right)=-\frac{v_{1}}{v_{2}}{ }_{B}
$$

Thus,

$$
\bar{\pi}(x) x \in \pi x(, 1)+(, 2)=\frac{1}{b-\delta a+},
$$

and the atoms are given by

$$
\pi[\& \delta, 2] b \delta C \frac{v_{1}}{\dot{v}_{2}}{ }_{B} \text { and }[, 1]=\quad{ }_{B}
$$

\subsubsection{One-Boundary Case}

Now, suppose that there is just the left boundary $a$, and the starting position of the process $x(t)$ is $x_{0} \in[a,+\infty)$. Then for $\mu>0$ we have the factor $C$, say $C_{O}$, given by

$$
C_{O}=\frac{v_{2} e^{\mu a}}{\frac{v_{1} v_{2}}{\lambda_{\underline{e}}}-\frac{\left(v_{1}-v_{2}\right)}{}} .
$$

Hence,

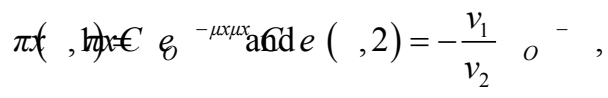

with the atom

$$
\pi \notin[, 2]=\frac{v_{1} v_{2}}{v_{1} v_{2}-\frac{\lambda_{2}\left({ }_{1} *{ }_{2}\right)}{\mu}}
$$

\section{Semi-Markov Case}

\subsection{Mathematical Model}

The particle movement is given by the equation

$$
x(t) \phi s x_{0} d s \cdot \int_{0}^{t} C(\quad())
$$

where $x_{0} \in[a, b]$ is the particle starting point inside the two reflecting boundaries $a<b$, and $\psi(\xi)$ is an alternating semi-Markov process with phase space $\mathbf{E}=\{1,2\}$ and embedded transition probability matrix $P$ given in (1). The sojourn time at state $i$ is a random variable with a common cumulative distribution function (cdf) $G_{i}(t), i \in \mathbf{E}$. We assume that $G_{1}(t)$ and $G_{2}(t)$ are not degenerated, and that their probability density function (pdf) and first moment, say

$$
g_{i}(t)=\frac{d G_{i}(t)}{d t} \text { and } m_{i}=\int_{0}^{\infty} \operatorname{tg}_{i}(t) d t \text { respectively, exist. }
$$

Now, the hazard rates are given by $r_{i}(t)=\frac{g_{i}(t)}{1-G_{i}(t)}$, and assume $C(1)=v_{1}>0$ and $C(2)=v_{2}<0$.

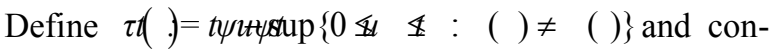
sider the three-component process $\chi(\tau t)=x(t(),()$, $\psi(())$ on the phase space $\mathbf{W}=[0, \infty) \times[a, b] \times\{1,2\}$. It is well-known that $\chi($ ) is a Markov process with the following infinitesimal operator $[8,9]$

$$
\begin{aligned}
& A \varphi(x, \dot{\varphi} \tau x) i=\frac{\partial}{\partial \tau}(,,)+ \\
& r_{i}(P \varphi)[\dot{\varphi} \dot{\varphi} \tau \mid l(\theta,, \varphi) \dot{x} \dot{x}(i,,)]+(,) \frac{\partial}{\partial x}(,,)
\end{aligned}
$$

with boundary conditions say $\frac{\partial \varphi(b,, 2)}{\partial \tau}=\frac{\partial \varphi(a,, 1)}{\partial \tau}$ $=0$ and $(\tau x i,) \in \mathbf{W}$. The function $\varphi(x, i$,$) is conti-$ nuously differentiable on $\tau$ and $x$. We also have that $P \varphi(\varphi, x, 1)=(0,, 2)$ and $P \varphi(0,, 2)=\varphi(0, ., 1)$

\subsection{Stationary Distribution}

Denote by $\rho(\cdot)$ the stationary distribution of the stochastic process $x(t)$. This stationary distribution has atoms at points $(\tau a, 2)$ and $(\tau b, 1)$, and we denote them as $\rho[a, 2]$ and $\rho \llbracket[b, 1]$, respectively. The continuous part of $\rho$ is denoted as $\rho(x, i),, i \in \mathbf{E}$.

For any function $\varphi(\cdot)$ belonging to the domain of the operator $A$ we have 


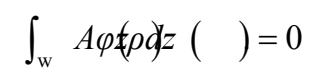

By changing the order of integration (integration by parts), we obtain expressions for $A^{*} \rho$, where $A^{*}$ is the adjoint operator of $A$, namely

$$
\left.\left.\frac{\partial}{\partial \tau x} \rho(x, i \rho, \tau) x\right) \operatorname{ti} \rho \tau x\right)(i,,) i+{ }_{i} \frac{\partial}{\partial}(,,)=0,=1,2,
$$

and

$$
\int_{0}^{\infty} r_{i} \tau(\tau) i(\tau d x, j) i=j i(9,,) ; \neq, \quad,=1,2,
$$

with the limiting behavior $\rho(t+\infty,)=$,0 , for all $x \in[a, b]$.

For the atoms we have

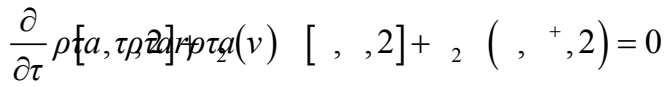

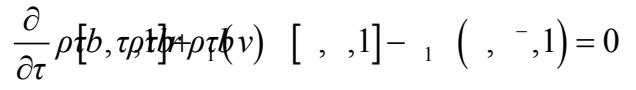

where

$$
\rho(b, \rho \bar{\tau} x, i):=\lim _{x \uparrow b}(,,) \text { and }
$$

$\rho\left(a, \rho \boldsymbol{\tau}^{\mathrm{x}}, i\right):=\lim _{x \downarrow a}(,$,$) , for i=1,2$. We also have

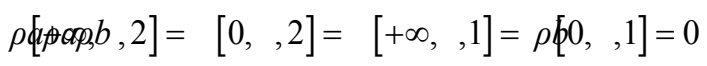

Now, by taking into account boundary conditions we have

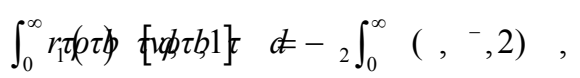

and

$$
\int_{0}^{\infty} r \tau(k \tau d) \tau[v d \tau a, 2 \tau] d={ }_{1} \int_{0}^{\infty}\left(,{ }^{+}, 1\right)
$$

By solving (27) we obtain

$$
\left.\rho(x, i, \partial) \operatorname{zrf} t x d l t v i_{i}\right) \exp \left(-\int_{0}^{\tau} i()\right),=1,2,
$$

where $f_{i} \in \mathcal{C}^{1}$.

By substituting (33) into (28) and by noting that

$$
\exp \left(-\int_{0}^{\tau} r_{i}(t) d t\right)=1-G_{i}()
$$

we obtain

$$
\int_{0}^{\infty} f_{i}(\operatorname{xg} \tau d \tau f) x(i) \quad j=i j_{j}(), . \neq, \quad=1,2
$$

It follows from (34) that

$$
\int_{0}^{\infty} \int_{0}^{\infty} f_{i}(x v-t i \tau \mathbf{g}-t d d t d) f\left(y ._{2}()={ }_{i}()\right.
$$

From (34) and (35) we can assume that the functions $f_{i}(x)$ are of the form

$$
f_{i}(x)=c_{i} e^{\lambda x}, i=1,2 .
$$

Now, by substituting (36) into (35), we obtain

$$
\hat{g} \lambda(\lambda){ }_{2}\left({ }_{2}\right)=1
$$

where $\hat{g}_{i}(s)=\int_{0}^{\infty} g_{i}(t) e^{-s t} d t$ is the Laplace transform of $g_{i}(t), i=1,2$. The set of pdf's for which (37) exists is similar to the set of functions which satisfies the Cramér condition.

Lemma 3.2.1 If $v_{1} m_{1}+v_{2} m_{2} \neq 0$, where $m_{1}=$ $\int_{0}^{\infty} \operatorname{tg}_{i}(t) d t$, and there exist $\ell_{1}<\ell_{2}, p_{1} \approx p_{2},{ }_{1}>0$ and $\sigma_{2}>0$ such that $\left.g_{1} \phi \boldsymbol{t}\right) \geq{ }_{1}, \in\left[\mathrm{g}, \ell_{t}\right],{ }_{2}() \geq \sigma_{2}$, $t \in\left[p_{1}, p_{2}\right]$ and $0<v_{1} \ell_{1}+v_{2} p_{2}, 0<-\left(v_{1} \ell_{1}+v_{2} p_{1}\right)$.

Then, there exists $\lambda_{0} \neq 0$ which satisfies (37).

Proof Let us define $p(g) \mid=\lambda \hat{g_{1}}\left(\begin{array}{l}1 \\ 1\end{array}\right){ }_{2}\left(\begin{array}{l}2 \\ 2\end{array}\right)$, so $p^{\prime}(0)=-\left(v_{1} m_{1}+v_{2} m_{2}\right) \neq 0$.

Now, suppose $p^{\prime}(0)=-\left(v_{1} m_{1}+v_{2} m_{2}\right)<0$. then

$$
p(e) \geq \oint_{\ell_{1}}^{\ell_{2}} t^{-v d t t^{2} \lambda t}{ }_{1}(e) \quad \oint_{p_{1}}^{p_{2}} t^{*} d t{ }_{2}(),
$$

hence

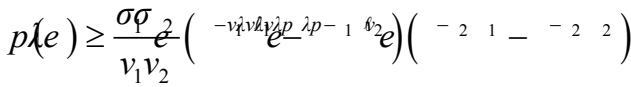

$$
\begin{aligned}
& \rightarrow+\infty \text { as } \lambda . \rightarrow+\infty
\end{aligned}
$$

The case $p^{\prime}(0)=-\left(v_{1} m_{1}+v_{2} m_{2}\right)>0$. can be reduced to the previous one by assuming $s=-t$ and using $0<v_{1} \ell_{2}+v_{2} p_{2}$.

\section{Theorem 3.2.1}

A)If $v_{1} m_{1}+v_{2} m_{2} \neq 0$ and $\lambda_{0} \neq 0$ is the solution for (37), and $\int_{0}^{\infty} e^{-\lambda_{t} \tau t} d \tau\left(1-G_{1}()\right)<+\infty$, then there exists a stationary distribution of $x(t)$ with the following continuous part:

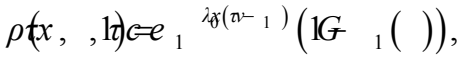

$$
\begin{aligned}
& \rho(x, \not 2 \mathcal{2}) \operatorname{ceg}_{1} \hat{1}_{1}\left(\begin{array}{ll}
0 & 1
\end{array}\right) G^{\not y(t i-2)}(1-\quad 2())
\end{aligned}
$$

and atoms

$$
\begin{aligned}
& \rho[\llbracket b, 1] \notin v_{1} e_{1}{ }^{\lambda}\left(1 G_{1}()\right) \frac{1-e^{-\lambda_{\theta} \tau_{1}}}{\lambda \gamma_{1}},
\end{aligned}
$$

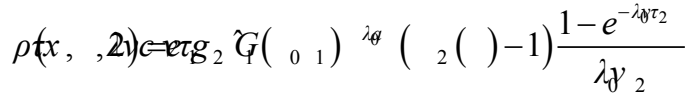

The normalization factor $c_{1}$ can be calculated from $\int_{\mathrm{w}} \rho(z)=.1$

B)If $v_{1} m_{1}+v_{2} m_{2}=0$ and there exists the second moment $\int_{0}^{\infty} t^{2} g_{i}(t) d t, i \in \mathbf{E}$, then the stationary measure of $x(t)$ is as follows 


$$
\rho(x,, z p) \approx{ }_{2}\left(1 G{ }_{1}(\not)\right),(,, 2)={ }_{2}\left(1-{ }_{2}()\right)
$$

with atoms

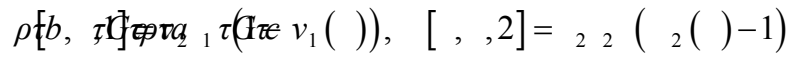

where

$$
c_{2}=\left[\left(m_{1}+m_{2}\right)(b-a)+v_{1} \frac{m_{1}^{(2)}}{2}-v_{2} \frac{m_{2}^{(2)}}{2}\right]^{-1} .
$$

Proof It is easy to see that $f_{1}(x)=c_{1} e^{\lambda_{0} x}$ and $f_{2}(x)=\mathscr{c}_{1} \hat{g}_{1}\left(\begin{array}{ll}0 & 1\end{array}\right)$ so satisfy (34). Substituting these functions $f_{i}$ into (33) we obtain (38) and (39). Therefore we substitute (38) and (39) into (29) and (30), then by solving these equations we obtain (40) and (41).

It can be easily verified that if $v_{1} m_{1}+v_{2} m_{2} \neq 0$ then the value $\lambda_{0} \neq 0$, such that $\hat{g} \lambda\left(\begin{array}{l}\gamma_{g} \\ g_{1}\end{array}\right){ }_{2}\left(\begin{array}{ll}0 & 2\end{array}\right)=1$, also satisfies (31) and (32).

Similarly, for $v_{1} m_{1}+v_{2} m_{2}=0$ we obtain (42) and (43) in the same manner as for the case $v_{1} m_{1}+v_{2} m_{2} \neq 0$ when it is considered that $\lambda_{0}=0$

We should notice that the stationary measure of the particle position $x(t)$ is determined by the following relations

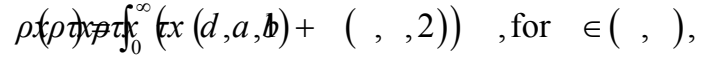

$$
\begin{aligned}
& \rho \notin \rho \tau \partial] \mp \oint_{0}^{\infty} \rho \tau b[\tau, d, 2],[, 1]=\int_{0}^{\infty}[,, 1]
\end{aligned}
$$

\section{Example Markov Case}

Suppose $g_{i}\left\langle(\notin) i={ }_{i}{ }^{-\lambda t} t,{ }_{i}>0,=1,2 ; \geq 0\right.$ Then,

$$
\hat{g} \lambda\left(\begin{array}{ll}
\lambda g \\
\gamma
\end{array}\right)_{2}\left(\begin{array}{ll}
0 & 2
\end{array}\right)=\left(\frac{\lambda \lambda}{\lambda \lambda+\lambda \lambda_{0} v_{1}}\right)\left(\frac{2}{2-a_{2}}\right)=1
$$

Therefore, $\lambda_{0}=\frac{\lambda \lambda}{v_{1}}+\frac{2}{v_{2}}$, and this case is the same as the one in the first part of this paper.

\section{Example Erlang Case}

Let $\quad g_{1}(\phi)=g \lambda \bar{p} t,{ }_{2} \lambda(t)={ }^{2}-p t,{ }_{1}>0,>0, \quad$ and $t \geq 0$. Then,

$$
\hat{g} \lambda\left(\begin{array}{ll}
\lambda & \lambda g
\end{array}\right)_{2}\left(\begin{array}{ll}
0 & 2
\end{array}\right)=\left(\frac{\lambda_{1}}{\lambda \lambda_{t} \lambda_{0} v_{1} p}\right)\left(\frac{p}{-0}\right)^{2}=1,
$$

where we have the conditions $\lambda \mu \nabla \lambda_{\nu} \gamma_{1}$ pnd $>_{02}$ Now, by solving (46) and taking into account the previous conditions, we obtain a unique solution for (46)

$$
\lambda_{0}=\frac{-\left(\lambda_{1} v_{2}+2 p \lambda p \lambda\right)+\sqrt{-4 v_{1} v_{2} p_{1}+\left({ }_{1}\right)^{2}}}{-2 v_{1} v_{2}}
$$

Since $r_{2}(t)=\frac{p^{2} t}{1+p t} \lambda \rightarrow p>0$, as $t \rightarrow+\infty$, and $r_{1}(t)={ }_{1}$, then the Theorem 3.2.1 is applicable.

\section{Conclusions}

The two-state continuous time random walk has been studied by many researchers for the Markov case and only a few have studied for non-Markovian processes [10]. This basic model has many applications in physics, biology, chemistry, and engineering. Most of the former models were oriented to solve the boundary-free particle motion. Recently this basic model has been extended in several directions, such as two and three dimensions, with reflecting and absorbing boundaries. Only a few of these works consider partly reflecting boundaries $[5,10]$, and references therein. However, in none of these previous works a stationary distribution for the particle position is presented, as we did in this paper. We have included the Markov case since it is illustrative and it motivates our analysis of the semi-Markov process.

\section{References}

[1] S. Goldstein, "On Diffusion by Discontinuous Movements and on the Telegraph Equation," The Quarterly Journal of Mechanics and Applied Mathematics, Vol. 4, No. 2, 1951, pp. 129-156.

[2] M. Kac, "A Stochastic Model Related to the Telegrapher's Equation," Rocky Mountain Journal of Mathematics, Vol. 4, No. 3, 1974, pp. 497-509.

[3] E. Orsingher, "Hyperbolic Equations Arising in Random Models," Stochastic Processes and their Applications, Vol. 21, No. 1, 1985, pp. 93-106.

[4] A. F. Turbin, "Mathematical Model of Einstein, Wiener, Levy," in Russian, Fractal Analysis and Related Fields, Vol. 2, 1998, pp. 47-60.

[5] J. Masoliver, J. M. Porrà, and G. H. Weiss, "Solution to the Telegrapher's Equation in the Presence of Reflecting and Partly Reflecting Boundaries," Physical Review E, Vol. 48, No. 2, 1993, pp. 939-944.

[6] V. S. Korolyuk and A. V. Swishchuk, A. V. Semi- Markov, "Random Evolutions," Kluwer Academic Publishers, 1995.

[7] V. S. Korolyuk and V. V. Korolyuk, "Stochastic Models of Systems," Kluwer Academic Publishers, 1999.

[8] V. S. Korolyuk and A. F. Turbin, "Mathematical Foundations of the State Lumping of Large Systems," Kluwer Academic Publishers, 1994.

[9] I. I. Gikhman and A. V. Skorokhod, "Theory of Stochastic Processes, Vol. 2," Springer-Verlag, New York, 1975.

[10] V. Balakrishnan, C. van den Broeck and P. Hangui, "First-Passage of Non-Markovian Processes: The Case of a Reflecting Boundary," Physical Review A, Vol. 38, No. 8, 1988, pp. 4213-4222. 